\title{
Validating benefit of biomarker-directed therapy for acute kidney injury: can you have your cake and eat it?
}

\author{
J. R. Prowle ${ }^{1,2^{*}}$ and M. Schetz ${ }^{3}$ \\ (c) 2017 Springer-Verlag Berlin Heidelberg and ESICM
}

Despite well-defined diagnostic criteria [1] there is currently little evidence that, beyond supportive care, targeted interventions for acute kidney injury (AKI) beneficially affect outcomes after AKI diagnosis. These failures can be explained by a time lag between the development of tubular injury, changes in GFR and the eventual rise in serum creatinine that signifies an AKI diagnosis, and also by the imprecise relationship between alteration in urine output and underlying AKI. As a result, renal pathology may be well established at the time of attempted intervention. In recognition of these limitations, over the last 15 years there has been a focus on the identification and characterization of plasma and urinary biomarkers for early renal injury [2]. However, efforts to validate and clinically apply these biomarkers have been hampered by the inadequate gold standard for current AKI diagnosis. Furthermore, proof of a statistical association with the development of AKI is very far from a demonstration that biomarker measurement will meaningfully alter physician treatment choices or clinical outcomes [3]. Consequently, until now there have been no positive reports of prospective randomized studies for biomarker-driven interventions for AKI, nor have any novel biomarkers been considered ready for incorporation into AKI diagnostic systems [4]. Given these concerns we have previously commented in this journal that, "prospective studies comparing outcomes from biomarker-directed care against conventional clinical and biochemical-directed intervention may be the only way to truly establish the

\footnotetext{
*Correspondence: j.prowle@qmul.ac.uk

${ }^{1}$ Adult Critical Care Unit and Department of Renal Medicine and Transplantation, The Royal London Hospital, Barts Health NHS Trust, Whitechapel Road, London E1 1BB, UK

Full author information is available at the end of the article
}

clinical value of novel (AKI) diagnostics" [5] and there is currently significant interest in how to incorporate renal biomarkers into interventional studies (Fig. 1) [6].

In this context, the recent prospective randomized trial by Meersch et al. [7] published in Intensive Care Medicine represents an important advance. It assesses a bundle of AKI-directed care in a population of at-risk patients identified by an elevated urinary AKI biomarker early after cardiac surgery. This study is important not so much for the actual findings or the specific content of the intervention but for the experimental approach, combining demonstration of both biomarker clinical utility and benefit of an early AKI intervention, two things that have been very difficult to prove in isolation.

In their study, Meersch et al. considered a population of patients undergoing cardiac surgery under cardiopulmonary bypass and randomized those identified as at higher risk of AKI by early elevation of a combination of two urinary cell cycle arrest markers (TIMP-2 and IGFBP-7) [8], above its lower, more sensitive, threshold [(TIMP-2) $\times($ IGFBP-7) $>0.3$ ]. Out of 882 eligible patients 276 were randomized. Biomarker-positive patients randomized to the treatment arm then received a bundle of AKI-directed care recommended in consensus treatment guidelines, involving haemodynamic optimization, the avoidance of nephrotoxins and maintenance of euglycaemia. Application of this bundle resulted in an absolute risk reduction of $16.6 \%$ (95\% CI 5.5-27.9\%) in the incidence of AKI within $72 \mathrm{~h}$ after surgery. Interestingly while a higher [TIMP-2] $\times$ [IGFBP-7] cut-off of $>2$ was highly predictive for development of AKI, in this group the intervention did not improve outcome, suggesting again that only patients with early, incipient, tubular injury may derive benefit from measures to prevent AKI.

\section{实 Springer}




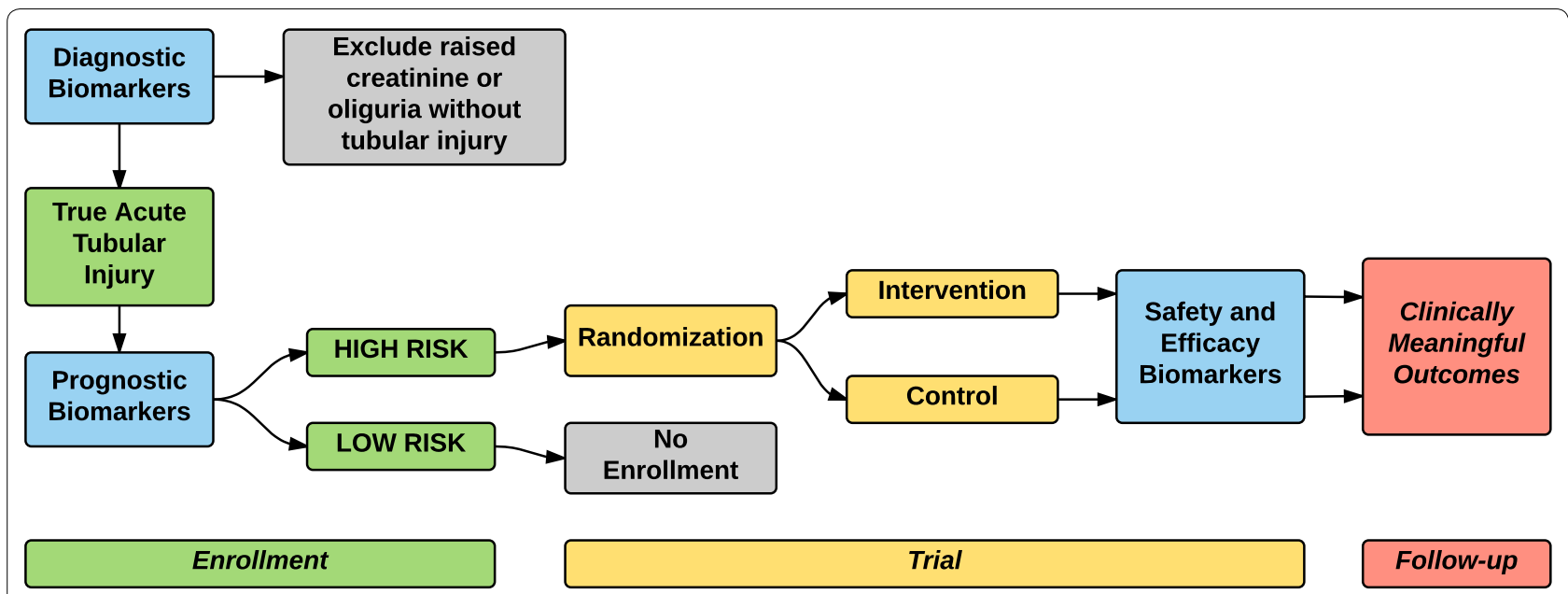

Fig. 1 Use of biomarkers in acute kidney injury trials. Diagnostic biomarkers can help enrol a subpopulation of patients with true tubular injury. Prognostic biomarkers can be used to enrol a subpopulation who are at greater risk of poor clinical outcomes including AKI progression. Safety and efficacy biomarkers can be used to assess AKI treatment effects that may be either beneficial or harmful Adapted from Parikh et al. [6]

Superficially these results appear very promising in supporting both the use of the biomarker and the benefit of an AKI treatment bundle. However, there are several important caveats to strong interpretation of these findings. The trial is unblinded and has been performed in a single-centre setting, which may limit the generalizability. The majority (more than $80 \%$ ) of AKI cases were diagnosed by KDIGO urine output rather than creatinine criteria. In fact, only eight patients had AK stage 2 or 3 by creatinine or both criteria. Although in a general ICU population oliguria is associated with patient-centred outcomes [9], this has not been shown in a cardiac surgery population. In this specific setting, it is plausible that haemodynamic interventions might influence the presence or absence of this criteria by simple physiological mechanisms without implication of overall benefit. This could explain why in the trial by Meersch et al. the intervention did not affect mortality, need for renal replacement therapy or kidney dysfunction at 30,60 or 90 days. There was even not a trend for a beneficial effect since most patient-centred outcomes were numerically higher in the intervention group. Whether a larger study could establish such an effect therefore remains questionable.

When evaluating any bundled intervention one must consider the content of the bundle and difference in uptake of the bundle components between groups to determine generalizability. In the study a greater use of inotropes (dobutamine), avoidance of ACE inhibitors/angiotensin receptor blockers (ARBs) immediately post-operatively and better avoidance of hyperglycaemia appear to be the major between-group differences. However, the bundle of interventions studied is recommended for patients with or at risk of early AKI and is not specifically tailored to the cardiac surgical population. It might be argued that interventions such as glucose control represent a standard of care for all cardiac surgical patients. Similarly, the choice regarding the continuation or cessation of ACE/ARBs around cardiac surgery remains contentious [10], and many clinicians would be cautious with these agents the day after surgery in a population where almost all patients required vasopressors at some point in the perioperative period. Finally, it is an important limitation that cardiac output monitoring was only used routinely in the intervention group so we cannot correlate any clinical effects of the haemodynamic interventions with any difference in outcome.

In conclusion, Meersch et al. have led the way in performing a positive study of a biomarker-directed intervention. The authors are cautious not to overinterpret their specific findings. As an unblinded single-centre study with a main outcome (AKI by oliguria) that is a questionable surrogate for long-lasting patient harm in the setting of cardiac surgery, these finding require confirmation in multicentred studies, powered to clinically relevant and patient-centred endpoints. Importantly, while some elements of the AKI bundle could be considered standards of care for all post-cardiac surgical patients, the value of a negative AKI biomarker in this context may be actually to identify a group of low-risk patients who can have normal regular medication and do not require invasive monitoring. Irrespective of the limitations in the specific clinical findings, this study represents the beginning of a new phase in renal biomarker research-away from collection of observational data-towards establishing the clinical utility of novel AKI biomarker measurements in prospective interventional studies. 


\section{Author details}

${ }^{1}$ Adult Critical Care Unit and Department of Renal Medicine and Transplantation, The Royal London Hospital, Barts Health NHS Trust, Whitechapel Road, London E1 1BB, UK. ${ }^{2}$ William Harvey Research Institute, Queen Mary University of London, London, UK. ${ }^{3}$ Division of Cellular and Molecular Medicine, Clinical Department and Laboratory of Intensive Care Medicine, KU Leuven University, Herestraat 49, 3000 Leuven, Belgium.

\section{Compliance with ethical standards}

\section{Conflicts of interest}

The authors declare no conflicts of interest.

Received: 31 January 2017 Accepted: 1 February 2017

Published online: 16 February 2017

\section{References}

1. Kidney Disease: Improving Global Outcomes (KDIGO) Acute Kidney Injury Work Group (2012) KDIGO clinical practice guideline for acute kidney injury. Kidney Int Suppl 2:1-138

2. Vanmassenhove J, Vanholder R, Nagler E, Van Biesen W (2013) Urinary and serum biomarkers for the diagnosis of acute kidney injury: an in-depth review of the literature. Nephrol Dial Transpl 28:254-273

3. Ostermann M, Joannidis M (2015) Biomarkers for AKI improve clinical practice: no. Intensive Care Med 41:618-622

4. McCullough PA, Shaw AD, Haase M, Bouchard J, Waikar SS, Siew ED, Murray PT, Mehta RL, Ronco C (2013) Diagnosis of acute kidney injury using functional and injury biomarkers: workgroup statements from the tenth acute dialysis quality initiative consensus conference. Contrib Nephrol 182:13-29
5. Prowle JR (2015) Measurement of AKI biomarkers in the ICU: still striving for appropriate clinical indications. Intensive Care Med 41:541-543

6. Parikh CR, Moledina DG, Coca SG, Thiessen-Philbrook HR, Garg AX (2016) Application of new acute kidney injury biomarkers in human randomized controlled trials. Kidney Int 89:1372-1379

7. Meersch M, Schmidt C, Hoffmeier A, Van Aken H, Wempe C, Gerss J, Zarbock A (2017) Prevention of cardiac surgery-associated AKI by implementing the KDIGO guidelines in high risk patients identified by biomarkers: the PrevAKI randomized controlled trial. Intensive Care Med. doi:10.1007/s00134-016-4670-3

8. Kashani K, Al-Khafaji A, Ardiles T, Artigas A, Bagshaw SM, Bell M, Bihorac A, Birkhahn R, Cely CM, Chawla LS, Davison DL, Feldkamp T, Forni LG, Gong MN, Gunnerson KJ, Haase M, Hackett J, Honore PM, Hoste EA, JoannesBoyau O, Joannidis M, Kim P, Koyner JL, Laskowitz DT, Lissauer ME, Marx G, McCullough PA, Mullaney S, Ostermann M, Rimmele T, Shapiro NI, Shaw AD, Shi J, Sprague AM, Vincent JL, Vinsonneau C, Wagner L, Walker MG, Wilkerson RG, Zacharowski K, Kellum JA (2013) Discovery and validation of cell cycle arrest biomarkers in human acute kidney injury. Crit Care 17:R25

9. Kellum JA, Sileanu FE, Murugan R, Lucko N, Shaw AD, Clermont G (2015) Classifying AKI by urine output versus serum creatinine level. J Am Soc Nephrol 26:2231-2238

10. Disque A, Neelankavil J (2016) Con: ACE inhibitors should be stopped prior to cardiovascular surgery. J Cardiothorac Vasc Anesth 30:820-822 